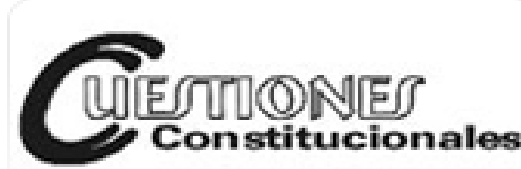

\section{Cuestiones Constitucionales}

ISSN: 1405-9193

rmdc@servidor.unam.mx

Universidad Nacional Autónoma de México

México

Méndez-Silva, Ricardo

Reformas a la Constitución Política en materia de tratados

Cuestiones Constitucionales, núm. 17, julio-diciembre, 2007, pp. 283-297

Universidad Nacional Autónoma de México

Distrito Federal, México

Disponible en: http://www.redalyc.org/articulo.oa?id=88501709

- Cómo citar el artículo

- Número completo

- Más información del artículo

Página de la revista en redalyc.org

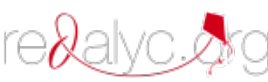

Sistema de Información Científica

Red de Revistas Científicas de América Latina, el Caribe, España y Portugal

Proyecto académico sin fines de lucro, desarrollado bajo la iniciativa de acceso abierto 


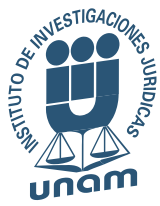

\title{
REFORMAS A LA CONSTITUCIÓN POLÍTICA EN MATERIA DE TRATADOS
}

\author{
Ricardo MÉNDEZ-SILVA*
}

Sin arrebatos se aprobó la reforma a los artículos constitucionales 76, fracción I y 89, fracción X, en las partes correspondientes a la participación del Senado de la República en la aprobación de los tratados celebrados por el Ejecutivo. La reforma apareció publicada en el Diario Oficial de la Federación el 12 de febrero de 2007.

El texto anterior contemplaba la participación del Senado únicamente en la aprobación de los tratados celebrados por el Ejecutivo. La novedad fue extender la autorización senatorial a las fases correspondientes a la terminación de los mismos, al retiro de las reservas, a la modificación y a la denuncia de los instrumentos convencionales.

Antes el artículo 76 establecía: "Son facultades exclusivas del Senado: I. Analizar la política exterior desarrollada por el Ejecutivo Federal con base en los informes anuales que el presidente de la República y el Secretario de Despacho correspondiente rindan al Congreso; además, aprobar los tratados internacionales y convenciones diplomáticas que celebre el Ejecutivo de la Unión". Con la reforma, hoy aparece de la siguiente manera:

Son facultades exclusivas del Senado:

Analizar la política exterior desarrollada por el Ejecutivo Federal con base en los informes anuales que el presidente de la República y el secretario del Despacho correspondiente rindan al Congreso

\footnotetext{
* Investigador en el Instituto de Investigaciones Jurídicas de la UNAM. Agradezco a mi asistente Mónica Cruz Espinosa su apoyo en la localización del material y la revisión de los borradores.
} 
Además, aprobar los tratados internacionales y convenciones diplomáticas que el Ejecutivo Federal suscriba, así como su decisión de terminar, denunciar, suspender, modificar, enmendar, retirar reservas y formular declaraciones interpretativas sobre los mismos;...

El artículo 76 había sido adicionado en 1977 cuando el Constituyente Permanente concedió al Senado la facultad de "analizar la política exterior". ${ }^{1}$ Eran tiempos de incipiente apertura política en el país cuando se despejó la participación de los partidos políticos en la Cámara de Diputados pero se reservó el delicado campo de la política exterior al conocimiento del Senado, Cámara que por mucho tiempo estuvo vedada al ingreso de miembros de la oposición política. El agregado tuvo una índole ambigua. La expresión "analizar" la política exterior parece revestida más bien de tintes académicos, reducida al terreno de la discusión y carente de correctivos formales al actuar del Ejecutivo en el plano internacional, aunque ciertamente la capacidad de maniobra política de la Cámara alta puede imprimirle un peso sustancial a esa facultad, etérea en la letra, de analizar la política exterior. Este punto permanece tal cual en la redacción presente.

A su vez el artículo 89, fracción X, fue adicionado en 1988 para incluir los principios que el presidente de la República debe seguir en la conducción de la política exterior. ${ }^{2}$ Hubo discusiones entonces sobre la conveniencia de concederle rango constitucional a los principios y sobre aquéllos que deberían ser reconocidos. Hasta el día de hoy, voces de la

1 “A partir de 1917 se le había otorgado como facultad exclusiva la de aprobar los tratados y las convenciones diplomáticas; sin embargo, con la reforma de 1977 a la fracción I del artículo, se consideró que dándole una atribución más genérica, se fortalecería al Senado en esta materia". Véase el comentario al artículo 76 constitucional de González Oropeza, Manuel y Ríos Estavillo, Juan José, Constitución Política de los Estados Unidos Mexicanos. Comentada, 5a. ed., México, PGR-UNAM, 1994, pp. 347-357.

2 Entonces se agregó: "En la conducción de tal política, el titular del Poder Ejecutivo observará los siguientes principios normativos: la autodeterminación de los pueblos; la no intervención; la solución pacífica de controversias; la proscripción de la amenaza o el uso de la fuerza en las relaciones internacionales; la igualdad jurídica de los Estados; la cooperación internacional para el desarrollo; y la lucha por la paz y la seguridad internacionales". Véase Méndez-Silva, Ricardo, "Bases constitucionales de la política exterior", en varios autores Problemas actuales del derecho constitucional. Estudios en homenaje a Jorge Carpizo, México, UNAM, Instituto de Investigaciones Jurídicas, 1994, pp. 253-275. 
reacción increpan esa reforma, sobre todo en lo concerniente a la prohibición de la intervención en los asuntos internos de otros países, a la solución pacífica de las controversias interestatales y a la proscripción del uso de la fuerza y de la amenaza en las relaciones internacionales. En buena medida estas desviaciones responden al propósito de alinear al país con la política exterior norteamericana tan propensa a los desplantes unilaterales. Recuérdese 2003, año en el que el gobierno del presidente W. Bush y su legión abyecta lanzaron su guerra de agresión contra Iraq. No pocos abogaban por que México se sumara o por lo menos manifestara su aquiescencia a esa guerra que ha devenido en un caos destructivo.

Dos motivaciones se develan en la reforma constitucional en comento. Primeramente, desde la perspectiva política, el intento de acotar las funciones del Ejecutivo tras los excesos que mostró durante décadas el desmesurado sistema presidencialista. Fueron tiempos en los que el Senado, facultado para la aprobación de los tratados, funcionó como una resonante caja de elogios, festejante de los dictados presidenciales. Desde el punto de vista técnico, las adiciones se entienden por la circunstancia de que en el Derecho Internacional y en la regulación interna la negociación y la firma de un tratado han correspondido tradicionalmente al Ejecutivo y la aprobación interna se ha encargado a un órgano legislativo, en nuestro caso al Senado, modelo inspirado en el sistema constitucional norteamericano. Pero ha persistido un vacío normativo respecto a la terminación de los tratados, al retiro de las reservas de exclusión o de interpretación, a la eventual modificación y a la posibilidad de denunciar un tratado cuando así lo contemple el régimen pactado.

A las claras, tratándose de la modificación de un tratado, el trámite debería ser el mismo que el de la conclusión original del acuerdo, es decir, debe prevalecer la participación del Senado. En 1998 México decidió aceptar la competencia obligatoria de la Corte Interamericana de Derechos Humanos. México era parte de la Convención Americana sobre Derechos Humanos pero no había aceptado la competencia obligatoria. Una vez que el país determinó integrarse al alto tribunal, la aceptación siguió 
los pasos de la aprobación interna del Senado en primera instancia y la declaración internacional respectiva a cargo del Ejecutivo.

Distinto es el caso de la denuncia, el acto jurídico que permite la terminación unilateral de un tratado. Conviene precisar lo suficiente que la denuncia sólo es aceptable jurídicamente cuando se encuentra prevista en el propio cuerpo convencional, ya que de otro modo la terminación unilateral sin el consentimiento de la o las contrapartes propiciaría el derrumbe de toda la mecánica convencional internacional. ${ }^{3}$ En nuestro tiempo son numerosos y muy importantes los tratados que incluyen una cláusula sobre denuncia en su articulado, bien a partir de un número determinado de años de vigencia del acuerdo, cinco, por ejemplo, o incluso en cualquier momento mediante el requisito de un preaviso que igual oscila entre seis meses o un año. En este supuesto la denuncia se encuentra aceptada en el texto del tratado y lógicamente es reconocida a través de la aprobación constitucional interna. Sin embargo, al presentarse la eventualidad de una denuncia conviene que junto a la determinación del Ejecutivo concurra la ponderación y la decisión del Senado.

Las iniciativas de reforma que se presentaron en el Senado ponían el acento en la denuncia. Las Comisiones Unidas de la Cámara que las estudiaron decidieron con buen tino incluir también a la terminación, ello en virtud de que la denuncia es un acto unilateral previamente pactado en el texto y la terminación opera a través del consentimiento de las partes cuando no se ha fijado un límite específico a su vigencia, sujeta, antes de esta reforma, a la determinación exclusiva del Ejecutivo. La desvinculación, sea de carácter unilateral, se insiste, cuando esté permitida, o bilateral o multilateral a posteriori, debe contar con la aprobación del Senado.

Las Comisiones Unidas del Senado incluyeron también la figura de las reservas. El voluntarismo estatal predominante en la contratación internacional motivó el nacimiento de esta figura ${ }^{4}$ en los tratados multilaterales, lo que permite a los Estados excluir la aplicación de un precepto o

3 "La denuncia es un acto que pone fin a la validez de una convención por la declaración unilateral que con tal fin hace una de las partes. La denuncia debe encontrarse prevista en la reconvención...". Kelsen, Hans, El contrato y el tratado. Analizados desde el punto de vista de la teoría pura del derecho, trad. de Eduardo García Máynez, México, Editora Nacional, 1974, p. 76.

4 Las reservas se pueden presentar durante la negociación, la firma, la ratificación o la adhesión. 
darle a alguno de los términos un sentido determinado. Existen dos tipos de reservas, de exclusión, que rechazan la aplicación de una X disposición, o interpretativa, que mantiene en vigor la disposición pero le confiere un sentido particular. En las adiciones constitucionales en comento se utiliza el término reservas para la exclusión y declaraciones interpretativas, se entiende, para las que le conceden un sentido específico a un término o a un artículo. Importa la participación del Senado en el retiro de una reserva por el efecto que tiene en el régimen de obligaciones y derechos originalmente pactados. Es hoy una preocupación toral del derecho internacional demandar a los Estados que retiren las reservas presentadas a los tratados, lo que asume importancia notoria en el campo de los derechos humanos y del derecho internacional humanitario, dados los valores y los bienes que defienden estas ramas de la materia. Es por tanto imprescindible que los Estados retiren sus objeciones y concedan vigencia plena a las convenciones sin parches excluyentes. Tiene el Senado en este punto un gran papel que cumplir.

El Senado conoció tres proyectos para la reforma que se aprobó el 12 de febrero de $2007 .{ }^{5}$

En primer lugar merece reconocimiento la senadora Gloria Lavara Mejía del Partido Verde Ecologista, quien presentó el 20 de marzo de 2003 una iniciativa de reforma a la fracción I del artículo 76 constitucional, sin que aludiera al 89, fracción X, introducido más tarde por las Comisiones Unidas del Senado. Es de destacarse igualmente que este proyecto se refirió a la denuncia de los tratados sin extenderse a las figuras de la terminación, modificación y reservas, que también fueron aportadas

5 El dictamen de las Comisiones Unidas puede consultarse bajo el siguiente título en la gaceta electrónica del Senado: "De las Comisiones Unidas de Puntos Constitucionales; de Relaciones Exteriores, Organismos Internacionales; de Estudios Legislativos; y de Estudios Legislativos, Primera, el que contiene proyecto de decreto por el que se reforma el artículo 76 fracción I; y el artículo 89 fracción X de la Constitución Política de los Estados Unidos Mexicanos", Gaceta del Senado, núm. 142, año 2005, martes 6 de diciembre, LIX Legislatura, 3er año de ejercicio, Primer Periodo Ordinario, http://www.senado.gob.mx/gaceta.php. 
en su momento por las Comisiones Unidas, como se señaló en el inciso anterior.

En el punto conducente, la iniciativa de la senadora Lavara Mejía propuso la adición al 76 constitucional, en los siguientes términos: “además, aprobar la celebración y la denuncia de los tratados internacionales y convenciones diplomáticas, que realice el Ejecutivo de la Unión".

Dos años más tarde, el 14 de abril de 2005, el senador priísta César Camacho Quiroz introdujo una iniciativa en la misma dirección, circunscrita igualmente al artículo 76, fracción I. Su propuesta trató dos puntos, el referente a la denuncia de los tratados y a la aprobación y denuncia, en su caso, de los acuerdos ejecutivos que la Ley sobre la Celebración de Tratados de 1992 bautizó como acuerdos interinstitucionales. ${ }^{6}$ Así las cosas, la propuesta rezaba: "además, aprobar o denunciar los tratados internacionales"; y se extendió a la necesidad de que el Senado participara en

6 En 1992 se aprobó la Ley sobre la Celebración de Tratados. Entonces opiné que no era un mayor logro legislativo se repetían definiciones y disposiciones de la Convención de Viena sobre el Derecho de los Tratados que ya para entonces era indiscutiblemente, con arreglo a lo dispuesto por el artículo 133, Ley Suprema de la Unión. Lo más preocupante es que la citada ley introdujo la figura de los "acuerdos interinstitucionales", no otra cosa que los acuerdos del Ejecutivo. Ciertamente la fluidez de las relaciones internacionales ha introducido en el derecho interno de los estados los acuerdos pactados exclusivamente por el Ejecutivo, pero en una ley secundaria se legalizaban acuerdos que no están contemplados por la carta magna. Las preocupantes deficiencias de esa Ley tienen todavía otras impericias. Los acuerdos interinstitucionales, carentes de constitucionalidad, se extendieron a acuerdos no solamente celebrados por el Ejecutivo a través de la Secretaría de Relaciones Exteriores sino a todas las dependencias del Ejecutivo, y se ampliaron incluso a los celebrados por los gobernadores de los estados y los presidentes municipales. La conducción de la política exterior del Estado corresponde al presidente de la República, tratándose de un Estado Federal lo que se encuentra asentado claramente en el texto supremo. Esta facultad genérica se correlaciona con la de celebrar tratados y con la participación del Senado en la aprobación respectiva. La Ley de 1992 atomiza esta facultad y desconoce la autoridad del Ejecutivo y del Senado y en la práctica da pie a una pluralidad de acuerdos que pueden contradecirse. La Ley impuso la obligación a las autoridades que celebraran este tipo de acuerdos a que los registraran en la Secretaría de Relaciones Exteriores pero ello se plantea a posteriori y no contempla la posibilidad de que sean dictaminados por la Cancillería. Por más de una razón es un régimen sin ton ni son que ya llega a los tres lustros de vigencia. Véase Méndez-Silva, Ricardo, "La ley de 1992 sobre la celebración de tratados", en varios autores, Modernización del derecho mexicano. Reformas constitucionales y legales 1992, México, UNAM, Instituto de Investigaciones Jurídicas, 1993, pp. 323-334. 
la aprobación y denuncia de los convenios ejecutivos y en general de los tratados de la siguiente manera: “...así como los acuerdos interinstitucionales, convenios ejecutivos, memoranda, convenciones, pactos, protocolos, entendimientos y convenciones diplomáticas que celebre el Ejecutivo de la Unión, cuando su naturaleza material tenga que ver con la seguridad nacional, inteligencia, fronteras, soberanía o cualquier otro tema que competa al interés esencial de la nación".

La iniciativa del Senador Camacho Quiroz se circunscribía a la adición de "denunciar los tratados internacionales" y puso el acento en la aprobación necesaria de los acuerdos ejecutivos por el Senado. Veamos lo tocante a éstos. En 1857 el Constituyente empleó la formula "tratados internacionales" y "convenciones diplomáticas" para cerrar la posibilidad de que el Ejecutivo celebrará acuerdos de cualquier tipo, leoninos al interés de la nación. El Constituyente tuvo en mente los acuerdos que se negociaron con otros Estados a fin de establecer las Comisiones de Reclamaciones por daños a extranjeros durante los desarreglos internos que tuvieron lugar durante el siglo XIX y el siguiente con los desastres de la época revolucionaria. ${ }^{7}$ El texto de 1857 fue trasladado a la carta constitucional de 1917 sin cambios. Por ello, todos los acuerdos, entendidos con arreglo a la terminología de la anterior carta liberal como los tratados internacionales y las convenciones diplomáticas deben pasar al conocimiento del Senado. ${ }^{8}$

Es ineludible reconocer que el dinamismo de la vida internacional obliga a la celebración de una gran cantidad de acuerdos informales que no necesariamente establecen normas de vigencia general y cuyos contenidos suelen ser de entidad menuda. La práctica los ha venido aceptando y sería una actitud burocrática involucrar al Senado en la aprobación de todos estos acuerdos pero al mismo tiempo sería irresponsable ignorar que hay acuerdos suscritos por el Ejecutivo, independientemente de su nombre, "entendimiento", "carta de intención", o el inventado por la Ley de 1992 de "acuerdos interinstitucionales", que llegan a establecer regí-

7 Ibidem, p. 331.

8 Originalmente la Constitución Política de 1857 fue unicameral y concedió la facultad de aprobar los tratados al Congreso. Cuando en 1874 se introdujo el Senado, fue a esta Cámara a la que se le encomendó tal facultad que pasó textual a la Constitución de 1917. Véase Méndez-Silva, Ricardo, op. cit., nota 2, p. 257. 
menes generales de carácter obligatorio. En tal situación no es posible consentir que se burle la aprobación del Senado.

La iniciativa del entonces senador César Camacho Quiroz se enfiló a prohibir en el texto constitucional la celebración de los acuerdos ejecutivos o interinstitucionales. La propuesta del prísta despierta algunos comentarios. La adición que se contemplaba en el artículo 76 constitucional rompía con la redacción de 1857, que con la frase "tratados internacionales y convenciones diplomáticas" cubría todo tipo de acuerdos. Son éstos los términos genéricos que adoptó la Constitución política. La Convención de Viena de 1969 sobre el Derecho de los Tratados adoptó el de tratado como término genérico, ${ }^{9}$ bajo el cual, con independencia de los nombres se ubica un conjunto de acuerdos, entre ellos los de protocolo, convención, pacto, e incluso el de los acuerdos ejecutivos, cuando estos se encuentran aceptados por el régimen jurídico interno. Por ello salía sobrando la enumeración particularizada que contemplaba esa iniciativa. Ahora bien, como los acuerdos ejecutivos no están previstos en nuestra carta magna sí era atendible el interés del legislador Camacho Quiroz de someter los acuerdos ejecutivos a la aprobación del Senado y en tal virtud resultaba procedente mencionarlos específicamente. ${ }^{10}$

La iniciativa del senador mexiquense, por su parte, proponía que pasaran al conocimiento del Senado para su aprobación los convenios cuyo contenido material tuviera que ver con la seguridad nacional, inteligencia, soberanía, fronteras o cualquier otro tema que involucrara el interés esencial de la nación. La expresión "cualquier otro tema que competa al interés esencial de la nación" suscita la siguiente interrogante: ¿conforme a qué criterios se solventaría la cuestión de si un tema es de interés esencial de la nación? Eso significa llanamente todos los tratados y consecuentemente no es necesario abundar en los casos o supuestos en los que es pertinente la aprobación del Senado. Bastaría con mencionar los trata-

9 La Convención de Viena de 1969 sobre el Derecho de los Tratados y Ley Suprema de la Unión señala que tratado es todo acuerdo celebrado por escrito, gobernado por el derecho Internacional, independientemente del nombre que reciba, esté contenido en uno o más instrumentos. Es decir bajo el término tratado se comprenden los de carta, pacto, convenio, acuerdo, protocolo y muchos más.

10 Es una cuestión que queda pendiente y debe afrontarse, diferenciándose aquéllos acuerdos de carácter estrictamente administrativo de los que establecen situaciones jurídicas generales. 
dos internacionales y las convenciones diplomáticas, y si acaso, como ya se dijo, los acuerdos ejecutivos.

El tercer proyecto fue presentado por el senador Adrián Alaniz Quiñones del Partido Revolucionario Institucional el 22 de septiembre de 2005 en consonancia con las dos iniciativas anteriores, centrado en la denuncia de los tratados. Su iniciativa no tocaba al ordenamiento constitucional y se ceñía a introducir enmiendas a la Ley sobre la Celebración de Tratados de 1992 y a la Ley sobre Aprobación de Tratados Internacionales en Materia Económica de 1994. En el artículo 5o. del primer instrumento preveía la redacción siguiente: "La voluntad de los Estados Unidos Mexicanos para obligarse por un tratado se manifestará a través de intercambio de notas diplomáticas, canje o depósito del instrumento de ratificación, adhesión o aceptación, mediante las cuales se notifique la aprobación o denuncia por el Senado del tratado en cuestión". Los artículos 9o. y 10 de la Ley sobre Aprobación de Tratados Internacionales en Materia Económica deberían quedar respectivamente con la redacción siguiente: "Para la aprobación o denuncia de algún tratado ya firmado deberá someterse al Senado junto con los siguientes documentos:... II. Una explicación de cómo la aprobación o denuncia del tratado afectará las leyes y reglamentos de México". Luego "Para la aprobación o denuncia a que se refiere el artículo anterior de la presente Ley, el Senado de la República, o en su caso la Comisión permanente deberán tunar el tratado a las comisiones competentes, en la sesión siguiente a la fecha en que el Ejecutivo Federal lo haya sometido al Senado".

Es nota distintiva de esta propuesta introducir la facultad del Senado en el tratamiento de las denuncias que promoviera el Ejecutivo a través de una ley secundaria. La preocupación era la misma, no dejarle las manos sueltas al presidente de la República, pero el problema de fondo era la falta de una previsión constitucional al respecto y, en un momento dado, la pretendida facultad del Senado hubiera quedado en entredicho.

IV

Las tres iniciativas pasaron a las Comisiones Unidas de Puntos Constitucionales, de Relaciones Exteriores, de Organismos Internacionales y de Estudios Legislativos del Senado. Fue en este espacio colegiado en donde se vinculó el artículo 76, fracción I, con el 89, fracción X. Ello por 
elementales razones de congruencia pues ambos preceptos se referían a la participación del Senado en la aprobación de los tratados. Y también junto a la obsesión de la denuncia, se añadieron las figuras de la terminación, la modificación y las reservas.

En la minuta disponible en Internet no se descubre la génesis de los cambios que condujeron a la redacción final. Están consignados, sin embargo, algunos puntos: "estimamos que es más adecuado, y con el afán de facilitar la comprensión de la norma constitucional, que la reforma comprenda al artículo 89, fracción X y al artículo 76, fracción I del texto constitucional". ${ }^{11}$ Fue en este espacio donde se confeccionó la versión definitiva de la reforma tal como fue finalmente aprobada. ${ }^{12}$

Prevaleció la coincidencia de opiniones en el Senado y realmente no se manifestaron oposiciones sobre los términos del Dictamen de las Comisiones Unidas. Intervinieron en el pleno a favor César Camacho Quiroz del Partido Revolucionario Institucional y Marco Antonio Adame Castillo del Partido Acción Nacional. La única discrepancia corrió a cargo del Senador Antonio Soto Sánchez del Partido de la Revolución Democrática, quien en el salón de sesiones de Xicotencatl tachó de sospechosa la moción de los otros partidos que buscaban impedirle al presidente de la República terminar, modificar o denunciar un tratado. Arguyó que la ampliación de las facultades del Senado en materia de política exterior había sido una antigua aspiración de su partido, mas mani-

11 Considerando número 6 del dictamen "De las Comisiones Unidas...", op. cit., nota 5.

12 A fin de facilitar la lectura se repite el texto de los artículos en estudio con las modificaciones aprobadas el 12 de febrero de 2007. El artículo 76 constitucional sobre las facultades exclusivas del Senado señala en su fracción primera:

"Analizar la política exterior desarrollada por el Ejecutivo Federal con base en los informes anuales que el presidente de la Republica y el Secretario del Despacho correspondiente rindan al Congreso.

Además, aprobar los tratados internacionales y convenciones diplomáticas que el Ejecutivo Federal suscriba, así como su decisión de terminar, denunciar, suspender, modificar, enmendar, retirar reservas y formular declaraciones interpretativas sobre los mismos;...".

Por su parte, el 89 sobre facultades y obligaciones del Presidente indica en su fracción décima:

"Dirigir la política exterior y celebrar tratados internacionales, así como terminar, denunciar, suspender, modificar, enmendar, retirar reservas y formular declaraciones interpretativas sobre los mismos, sometiéndolos a la aprobación del Senado;...”, cursivas del autor que destacan las adiciones. 
festó su extrañeza de que en ese momento se intentara recortar la capacidad de acción del Ejecutivo Federal en los asuntos internacionales: "Es una vieja demanda que se puede, el día de hoy, concretar. Sin embargo nos parece altamente sospechoso lo que están haciendo". "La senadora Lavara Mejía había introducido el tema en 2003 pero ya corría el 2005 y las encuestas electorales marcaban una holgada preferencia de la opinión pública a favor del entonces jefe de Gobierno del Distrito Federal para la Presidencia de la República, quien por su ideario político no parecía simpatizar, entre otros instrumentos, con el Tratado de Libre Comercio de América del Norte. La obsesión de impedir la denuncia por el Ejecutivo de los tratados en las tres iniciativas sí da a pensar. Con todo, desde la perspectiva elemental, verdaderamente elemental, del derecho de los tratados, no hubiera tenido cabida legal una denuncia, pues sólo es valedera si se encuentra prevista en el texto del tratado, cosa que no ocurre con el TLCAN. Las preocupaciones, si las hubo entre los promotores de la reforma, orbitaban en torno al suceder interno pero el derecho Internacional existe y goza de cabal salud a pesar de los avatares que padece. En la misma sesión, Jorge Zermeño Infante, senador panista, alivió las inquietudes del senador Soto Sánchez. Y hubo final feliz, en tiempos de duro forcejeo parlamentario votaron 105 senadores a favor sin voto en contra. ¡Unanimidad!, proclamó la secretaria de la Cámara de Senadores.

\section{V}

El 6 de diciembre de 2005, el mismo día en el que la Cámara de origen aprobó la iniciativa, envió a la Cámara de Diputados la Minuta Proyecto de Decreto de los artículos 76 y 89 constitucionales. Dos días después la Minuta fue turnada a la Comisión de Puntos Constitucionales para que la dictaminara y a la Comisión de Relaciones Exteriores a efecto de que emitiera su opinión.

La Comisión de Puntos Constitucionales estimó que la denuncia de un tratado debe ser un acto coordinado entre los Poderes Ejecutivo y Legis-

13 Puede consultarse la trascripción de la discusión realizada sobre este dictamen así como los resultados de la votación en el Diario de los Debates, núm. 31, del 6 de diciembre de 2005, Legislatura LIX, Año III, Primer Periodo Ordinario, http://www.sena do.gob.mx/diario.php. 
lativo, "en el que el Ejecutivo proponga y el Legislativo disponga", ${ }^{14}$ frase célebre acuñada en tiempos ya idos. Y agregó con nitidez: "La denuncia de cualquier instrumento internacional debe formar parte de las facultades expresas del Senado". Fue un dictamen pulcro con elaboraciones interesantes que incluyen material de fuentes académicas sin que, por cierto, se hagan las citas correspondientes a pie de página. La Comisión aprobó en sus términos, sin cambios ni ajustes, el 5 de abril de 2006 la Minuta remitida por el Senado de la República y presentó al pleno el Proyecto de Decreto de reforma constitucional. La Cámara de Diputados en votación económica dispensó la lectura del Dictamen y sin discusión fue aprobado el 25 de abril con votación de consenso, aunque, salta a la vista, con un buen número de representantes ausentes: 352 votos a favor y dos abstenciones, una de un prísta y otra de un perredista. ${ }^{15}$

El 11 de enero de 2007 la Cámara de Diputados envió a la Comisión Permanente del Congreso de la Unión la relación de veinte aprobaciones por sendas legislaturas de los estados. ${ }^{16}$ La Comisión Permanente formuló la Declaratoria de Reformas Constitucionales el 17 de enero siguiente y la turnó al presidente de la República para sus efectos constitucionales. ${ }^{17}$

Correspondió al nuevo titular del Ejecutivo Federal, Felipe de Jesús Calderón Hinojosa, publicar el mencionado Decreto en el Diario Oficial el 12 de febrero, habiendo entrado en vigor al día siguiente. Se estrenó el nuevo mandatario con esta reforma que tiene una clara dedicatoria al presidente de la República.

14 Puede consultarse el texto del dictamen bajo el título "De la Comisión de Puntos Constitucionales, con proyecto de decreto que reforma los artículos 76, fracción I; y 89, fracción X, de la Constitución Política de los Estados Unidos Mexicanos", Gaceta Parlamentaria, año IX, núm. 1991, jueves 20 de abril de 2006, http://gaceta.diputados.gob.mx/.

15 Idem.

16 Aguascalientes, Baja California, Baja California Sur, Campeche, Colima, Chiapas, Chihuahua, Guanajuato, Hidalgo, Morelos, Oaxaca, Puebla, Quintana Roo, San Luis Potosí, Sinaloa, Sonora, Tabasco, Tamaulipas, Veracruz y Zacatecas.

17 Comisión Permanente, "Resumen de la sesión de la Comisión Permanente celebrada el miércoles 17 de enero de 2007", Primer Receso, Primer Año de Ejercicio, p. 3, http://archivos.diputados.gob.mx/servicios/datorele/LX_LEG/PERMANENTE/17-ene-07/ 17-ene-07.pdf. 
Para concluir, unos apuntes finales. Las reformas constitucionales corresponden a la tendencia de acotar las atribuciones del Ejecutivo que prevalecieron en la época del presidencialismo exacerbado en México. Poco o nada influía el Senado en la revisión efectiva de los tratados celebrados antaño por el presidente de la República.

Las bases normativas con las que cuenta el Senado deben ser el cimiento de una actuación responsable en materia de política exterior. Fue lamentable, hay que decirlo, la reforma al artículo 21 constitucional sobre el Estatuto de la Corte Penal Internacional. ${ }^{18}$ En nada honra a la tradición internacionalista de México ese disparate jurídico. ${ }^{19}$ Vale señalar que la reforma promovida y aprobada equivale a una reserva encubierta no obstante que el Estatuto impide la presentación de reservas. ${ }^{20}$ Aflora persistente una visión arcaica de la soberanía y el texto termina emparentándose con los intentos de los Estados Unidos de minar al Tribunal de La Haya.

La ampliación de las facultades del Senado debe mover a un ejercicio responsable de las mismas. Mal estaría que se aprovecharan para oponer escollos burocráticos, especialmente en el retiro necesario de las reservas que México ha presentado en materia de derechos humanos. Podría el Senado abocarse a estudiar la pertinencia de retirar si no todas, algunas de ellas. ${ }^{21}$

18 Párrafo quinto del artículo 21 constitucional: "El Ejecutivo Federal podrá, con la aprobación del Senado en cada caso, reconocer la jurisdicción de la Corte Penal Internacional". Adicionado mediante Decreto publicado en el Diario Oficial de la Federación el 20 de junio de 2005.

19 Véase García Ramírez, Sergio, La Corte Penal Internacional, 2a. ed., México, INACIPE, pp. 151-165; y Becerra Ramírez, Manuel, "México ratifica el Estatuto de Roma que crea la Corte Penal Internacional, después de reformar la Constitución", Anuario Mexicano de Derecho Internacional, México, vol. VI, 2006, pp. 951-954.

20 El artículo 120 del Estatuto de Roma de la Corte Penal Internacional aprobado el 17 de julio de 1998 señala claramente: "No se admitirán reservas al presente Estatuto".

21 Véase la tercera conclusión de la Mesa 3 "Derecho Internacional de los Derechos Humanos" del VII Congreso Iberoamericano de Derecho Constitucional: "Promover la adopción de los principios de universalidad de los tratados relativos a los derechos humanos y de eliminación de las reservas, así como el fortalecimiento de las instancias judiciales y cuasijudiciales de los citados derechos", en Méndez-Silva, Ricardo (coord.), Derecho Internacional de los Derechos Humanos. Memoria del VII Congreso Iberoamericano 
Pervive en la Constitución Política el tristemente célebre artículo 33 constitucional que faculta al presidente de la República a expulsar discrecionalmente a los extranjeros cuya permanencia dentro del territorio nacional considere indeseable. Aun cuando se incluyó la disposición en la Constitución de 1917 hubo entonces una polémica airada. El precepto respondió a las turbulencias de la época revolucionaria pero carece de justificación en las circunstancias presentes y va en contra de los tratados fundamentales de derechos humanos. México al suscribir tratados como el Estatuto de los Refugiados de 1951 o al haber aceptado la competencia obligatoria de la Corte Interamericana de Derechos Humanos ha presentado reservas a fin de salvar la oposición entre el artículo 33 y los instrumentos internacionales mencionados.

Las reformas se alcanzaron con facilidad porque no se tocaron cuestiones políticas delicadas o aspectos técnicos enredados. Quedan, sin embargo, algunos puntos que deben atenderse en materia de tratados, entre ellos el de los acuerdos interinstitucionales o ejecutivos.

En una reforma a fondo del Estado deberá discutirse la participación de la Cámara de Diputados en la aprobación de los tratados, sobre todo en aquellas materias en las que la Constitución Política indica que son facultad del Congreso. ${ }^{22}$ En la regulación actual, en la celebración de un tratado que verse sobre esas materias, intervienen únicamente el Ejecutivo y el Senado y no obstante tienen el rango de ley suprema de la Unión en un nivel superior al de las leyes emanadas de la Constitución como lo ha determinado la Suprema Corte de Justicia de la Nación en dos ocasiones.

de Derecho Constitucional, México, UNAM, Instituto de Investigaciones Jurídicas, 2002, p. 690.

22 El profesor Antonio La Pergola hizo notar desde hace tiempo las complicaciones que pueden surgir de un régimen dual de elaboración de leyes. Véase de este autor Constitución del Estado y normas internacionales, México, UNAM, 1985, pp. 25 y ss. También el embajador Palacios Treviño se ha pronunciado por la participación de la Cámara de Diputados en la celebración de tratados sobre materias que la Constitución Política considera competencia del Congreso de la Unión. Véase Palacios Treviño, Jorge, Tratados, legislación y práctica en México, México, Secretaria de Relaciones Exteriores, 1982, p. 16. Más recientemente Diego Valadés ha sostenido una postura idéntica, y es también mi opinión. Véase respectivamente Valadés, Diego, "Asimetrías en el Congreso", Excélsior, México, 27 de marzo de 2000; y Méndez-Silva, Ricardo, "La celebración de los tratados, genealogía y actualidad constitucional”, Anuario Mexicano de Derecho Internacional, México, vol. I, 2001, pp. 291-322. 
En consecuencia, debe abordarse lo tocante a la jerarquía de los tratados en nuestro sistema constitucional. La Suprema Corte de Justicia de la Nación en una Tesis del 28 de octubre de $1999,{ }^{23}$ reiterada en otro asunto el 13 de febrero de 2007, ha señalado que los tratados se encuentran por encima de las leyes emanadas de la Constitución y por debajo de ésta. Concuerdo plenamente con los pronunciamientos del alto tribunal, aunque es menester advertir que no hay asidero jurídico para tal conclusión ni dentro del texto constitucional ni en los trabajos preparatorios del Constituyente, por lo que la prudencia aconsejaría que el legislador validara de modo inobjetable las tesis de referencia.

En el mismo tenor de la jerarquía de los tratados dentro de nuestro sistema jurídico hay otro asunto pendiente. Han irrumpido en el mundo del derecho las Constituciones internacionalistas, una nueva generación de leyes fundamentales que conceden rango superior a las normas convencionales internacionales, objeto de estudio por el maestro Héctor Fix-Zamudio. ${ }^{24}$ La tendencia dominante del constitucionalismo moderno camina hacia esta dirección, de hecho, la Convención de Viena sobre el Derecho de los Tratados, ratificada por México, tiene una disposición al respecto: No se puede anular un tratado con base en una ley interna. ${ }^{25}$ Sin embargo, creo en términos realistas que el medio político y aún una parte del jurídico no están preparados todavía para dar ese salto audaz en el corto plazo pero estoy convencido de que el punto debe quedar inscrito responsablemente en la agenda de las discusiones y ser considerado, al menos en principio, en lo que toca a los tratados de derechos humanos que deben elevarse a una escala predominante con respecto a las Constituciones.

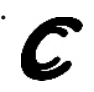

23 Véase Becerra Ramírez, Manuel et al., "Tratados internacionales. Se ubican jerárquicamente por encima de las leyes y en un segundo plano respecto de la Constitución Federal (Amparo en revisión 1475/98)", Cuestiones Constitucionales, México, núm. 3, julio-diciembre de 2000, pp. 169-208.

24 Véase Fix-Zamudio, Héctor, La Constitución y su defensa, México, UNAM, 1984, pp. 77 y ss.

25 Artículo 27 de la Convención de Viena sobre el Derecho de los Tratados de 1969: "Una parte no podrá invocar las disposiciones de su derecho interno como justificación del incumplimiento de un tratado. Esta norma se entenderá sin perjuicio de lo dispuesto en el artículo 46". 